This section of Epidemiology and Psychiatric Sciences appears in each issue of the Journal to stress the role of the epidemiological approach to promote advances in the field of clinical psychopharmacology, with a particular attention to controversial findings. The ultimate aims are to help develop a more critical attitude towards the results of research studies published in the international literature, to promote original research projects with higher methodological standards, and to implement the most relevant results of research in every-day clinical practice. These contributions are written in house by the journal's editorial team or commissioned by the Section Editor (no more than 1000 words, short unstructured abstract, four key-words, one Table or Figure and up to ten references).

Corrado Barbui, Section Editor

\title{
Antidepressant dose and the risk of deliberate self-harm
}

\author{
C. Barbui ${ }^{1 *}$ and S.B. Patten ${ }^{2}$ \\ ${ }^{1}$ Department of Public Health and Community Medicine, Section of Psychiatry, University of Verona, Verona, Italy \\ ${ }^{2}$ Department of Community Health Sciences, Faculty of Medicine, University of Calgary, Calgary, Alberta, Canada
}

\begin{abstract}
Although the mechanism by which antidepressants (ADs) may increase the risk of suicide-related outcomes is unknown, it has been hypothesised that some adverse effects, including akathisia, insomnia and panic attacks, as well as an early energising effect that might allow patients with depression to act on suicidal impulses, may have a key role. Considering that these adverse effects are dose-related, it might be hypothesised that the risk of suicidal behaviour is similarly related to the AD dose. This research question has recently been addressed by a propensity scorematched observational cohort study that involved 162625 patients aged 10-64 years with a depression diagnosis who initiated therapy with citalopram, sertraline or fluoxetine. In this commentary, we discuss the main findings of this study in view of its methodological strengths and limitations, and we suggest possible implications for day-today clinical practice.
\end{abstract}

Received 26 May 2014; Accepted 12 June 2014; First published online 14 July 2014

Key words: Adults, deliberate self-harm, dose, selective serotonin reuptake inhibitors, suicide, youth.

In May 2007 the Food and Drug Administration (FDA) ordered that all antidepressant (AD) drugs should carry an expanded black-box warning incorporating information about an increased risk of suicidal symptoms in young adults aged 18-24 years. The warning was based on the results of a FDA meta-analysis that included 372 placebo-controlled AD trials and nearly 100000 patients (Stone et al. 2009). On the basis of this analysis the relationship between $\mathrm{AD}$ treatment and the incidence of reported suicidal behaviour in

* Address for correspondence: Professor Corrado Barbui, Department of Public Health and Community Medicine, Section of Psychiatry, University of Verona, Piazzale L.A. Scuro 10, 37134 Verona, Italy.

(Email: corrado.barbui@univr.it) clinical trials was strongly related to age: the risk associated with drug treatment relative to placebo was found to be elevated in subjects under age 25, neutral in subjects aged 25-64 (reduced if suicidal behaviour and ideation are considered together) and reduced in subjects aged 65 and older. Another FDA meta-analysis suggested that the rate of suicidal ideation and behaviour in children randomised to $\mathrm{AD}$ drugs was twice as compared with children randomised to placebo (Hammad et al. 2006).

Although the mechanism by which AD use may increase the risk of suicide is unknown, it has been hypothesised that some adverse effects, including akathisia, insomnia and panic attacks, as well as an early energising effect that might allow patients with depression to act on suicidal impulses, may have a 
key role. Considering that these adverse effects are dose-related, it might be hypothesised that the risk of suicidal behaviour is similarly related to AD dose. This research question has recently been addressed by Miller and colleagues, who carried out a propensity score-matched cohort study, based on observational health care utilisation data (Miller et al. 2014).

The study involved 162625 patients aged 10-64 years with a depression diagnosis who initiated therapy with citalopram, sertraline or fluoxetine. According to the $\mathrm{AD}$ dose prescribed among the AD initiators, patients were assigned to a modal-dose category or to a highdose category. The modal daily dose for citalopram, sertraline and fluoxetine were, respectively, 20, 50 and 20 $\mathrm{mg} /$ day. Study follow-up began the day after initiation of the first AD therapy, and the outcome of interest was the first occurrence of acts of deliberate self-harm (DSH). Interestingly, patients were divided into two age groups guided by the age-related risk of suicidal behaviour identified in the FDA meta-analyses: ages 10-24 years v. 25-64 years. The study found that the rate of DSH in the 10-24 age group who initiated highdose therapy was approximately twice as high as among the matched patients initiating modal-dose therapy (hazard ratio 2.2, 95\% confidence interval from 1.6 to 3.0). By contrast, no effect was detected in the 25-64 age group.

As suggested by Brent and Gibbons in an accompanying commentary (Brent \& Gibbons, 2014), this pharmacoepidemiological study has several methodological strengths, including avoiding the use of a comparison group of individuals who were not exposed to $\mathrm{AD}$, which would have lead to a risk of confounding by indication, and the use of propensity score matching, which allowed to produce groups that were well balanced in terms of observed potential confounders. Another strength is that suicidal events, a commonly used composite suicide measure that lumps together suicide ideas and thought with suicide attempts, was not employed as outcome measure.

As with all observational studies, there are a number of considerations that might be raised when interpreting these findings. First, the outcome of interest, acts of DSH, is without any doubt a clinically relevant pragmatic measure, but it is nevertheless true that the majority of individuals who deliberately commit acts of self-harm do not commit suicide. According to a recent meta-analysis, the incidence of suicide in individuals presenting to hospital for self-harm is $1.6 \%$ at 1 year, $2.1 \%$ at 2 years, $3.9 \%$ at 5 years and $4.2 \%$ at 10 years (Carroll et al. 2014). The number needed to harm for suicide can therefore be expected to be much higher than those reported for DSH. Clinically, therefore, it would be of interest to know if the findings of the present study may be replicated employing completed suicide, rather than DSH, as an outcome measure. We argue that a sensitivity analysis with completed suicide, even if statistically unpowered, would have provided clinicians with an initial interesting insight into this compelling issue. The negative implications of conducting such an analysis (the risk of Type II error in an underpowered analysis) could be minimised by presenting confidence intervals for the hazard ratio. A second clinical reasoning on DSH is that this is a typical psychopathological feature of borderline personality traits, which are common in depressed individuals (Bagby et al. 2008). Clinical guidelines suggest, when approaching a depressed patient, to carefully consider borderline personality traits, such as impulsivity that may have a strong independent impact on suicidality. One might argue, therefore, that this study may have at least partially captured DSH as a consequence of impulsivity linked to borderline personality traits, rather than suicidality as a consequence of adverse effects of $\mathrm{AD}$ exposure. This patient population might be particularly vulnerable to high-dose AD therapy through other mechanisms, such that such traits may modify as well as confound the observed association.

Another interesting finding is that nearly $20 \%$ of the population initiated treatment with high-dose AD. High-dose AD treatment may be a proxy of severity of depression, or may identify patients with previous suicide ideas, or patients who failed to respond to standard AD dose. In any case, it may identify a population at a greater risk for DSH for reasons other than AD dose. The extent to which the propensity matching was successful in controlling such factors cannot be known with certainty, such that independent replication of these results will be important. According to the analysis carried out by the study authors, predictors of initiating therapy with high- rather than modal-dose AD included, having been admitted to a psychiatric hospital in the year prior to starting AD therapy, having an internist (rather than a psychiatrist or other health professional) prescribe the initial $\mathrm{AD}$, taking no prescription medications other than the AD initiated, and being prescribed sertraline rather than fluoxetine or citalopram. Patients in the high-dose group, therefore, may be at greater risk for DSH.

Conversely, in the modal-dose group, that is patients who initiated citalopram at $20 \mathrm{mg} /$ day, sertraline at 50 $\mathrm{mg} /$ day and fluoxetine $20 \mathrm{mg} /$ day, data on those who needed a change in dose, for example a dose increase, or to switch or add $\mathrm{AD}$, were censored. One may argue that these strategies, commonly employed under ordinary circumstances, identify patients who did not perform well with the initial modal dose that is patients who might be more severely ill and therefore at greater risk for DSH. Censoring their data, therefore, might have 
systematically excluded the severe cases from the modal-dose group, diminishing the denominator of the hazard ratio and thereby increasing the ratio itself. Similarly, cautious clinicians may have initiated treatment at low dosages in patients they believed to be at high risk of DSH while nevertheless having the intention of later increasing their dosage to the modal dose. These patients were excluded from the analysis, potentially removing some high-risk patients from the modal-dose cohort.

Although this study showed that a possible unmeasured confounder would have to be larger than the effect of prior DSH on future DSH, clinical reasoning would suggest that the two cohorts may have subtle but clinically relevant differences that could not be easily added to the long and comprehensive list of confounders that were included in the statistical analysis. The most effective method of controlling for confounding, including unmeasured confounders, is randomisation. Meta-analyses of DSH data from studies randomly assigning subjects to different dosages would be welcome additions to the literature.

Finally, the following are subgroup analyses that, ideally, would have been clinically interesting. First, grouping individuals below 25 years of age into subgroups, as a young adult aged 24 is clinically different from an adolescent of 12 years; second, stratifying the analysis by sex, as data have shown that the occurrence of suicide-related outcomes is substantially different in males as compared with females; third, stratifying the analysis by $\mathrm{AD}$ drug, as data have shown that there may be substantial differences among AD drugs.

In summary, this thoughtful analysis of the effects of initiating AD therapy at higher than modal doses is a major epidemiological achievement. The finding that high initial $\mathrm{AD}$ dose leads to an increased risk for DSH is a new, original finding that has substantial implications for everyday clinical practice, as it clearly points out that AD treatment should not be started with greater than modal doses. This study, however, does not address the issue of dose change or dose escalation.

\section{Financial support}

No financial support was received for this paper from any funding agency, commercial or not-for-profit sectors.

\section{Conflict of interest}

None.

\section{References}

Bagby RM, Psych C, Quilty LC, Ryder AC (2008). Personality and depression. Canadian Journal of Psychiatry 53, 14-25.

Brent DA, Gibbons R (2014). Initial dose of antidepressant and suicidal behavior in youth: start low, go slow. JAMA Internal Medicine, in press.

Carroll R, Metcalfe C, Gunnell D (2014). Hospital presenting self-harm and risk of fatal and non-fatal repetition: systematic review and meta-analysis. PLOS ONE 9, e89944.

Hammad TA, Laughren T, Racoosin J (2006). Suicidality in pediatric patients treated with antidepressant drugs. Archives of General Psychiatry 63, 332-339.

Miller M, Swanson SA, Azrael D, Pate V, Sturmer T (2014). Antidepressant dose, age, and the risk of deliberate self-harm. JAMA Internal Medicine, in press.

Stone M, Laughren T, Jones L, Levenson M, Holland PC, Hughes A, Hammad TA, Temple R, Rochester G (2009). Risk of suicidality in clinical trials of antidepressants in adults: analysis of proprietary data submitted to US Food and Drug Administration. BMJ 339, doi: 10.1136/bmj. b2880. 